\title{
THE DARK ENERGY SURVEY CCD IMAGER DESIGN
}

\author{
H. Cease ${ }^{* a}$, D. DePoy ${ }^{\mathrm{b}}$ G. Derylo ${ }^{\mathrm{a}}$, H. T. Diehl ${ }^{\mathrm{a}}$, J. Estrada ${ }^{\mathrm{a}}$, B. Flaugher ${ }^{\mathrm{a}}$, V. Guarino ${ }^{\mathrm{c}}$ K. Kuk ${ }^{\mathrm{a}}$, \\ S. Kuhlmann ${ }^{\mathrm{c}}$, K. Schultz , R.L. Schmitt ${ }^{\mathrm{a}}$ A. Stefanik ${ }^{\mathrm{a}}$, A. Zhao ${ }^{\mathrm{c}}$ \\ ${ }^{\mathrm{a}}$ Fermi National Accelerator Laboratory ${ }^{1}$, Box 500, Batavia, IL, USA 60510 \\ ${ }^{\mathrm{b}}$ Ohio State University, ${ }^{\mathrm{c}}$ Argonne National Laboratory, Argonne, IL, USA 60439
}

FOR THE DARK ENERGY SURVEY COLLABORATION

\begin{abstract}
The Dark Energy Survey is planning to use a 3 sq. deg. camera that houses a $\sim 0.5 \mathrm{~m}$ diameter focal plane of $622 \mathrm{kx} 4 \mathrm{k}$ CCDs. The camera vessel including the optical window cell, focal plate, focal plate mounts, cooling system and thermal controls is described. As part of the development of the mechanical and cooling design, a full scale prototype camera vessel has been constructed and is now being used for multi-CCD readout tests. Results from this prototype camera are described.
\end{abstract}

Keywords: Dark Energy, CCD, imager, design, Blanco, CTIO, Cooling

\section{INTRODUCTION}

The Dark Energy Camera (DECam), ${ }^{1}$ will be the primary instrument used in the Dark Energy Survey ${ }^{2}$. DECam will be a 3 sq. deg. mosaic camera mounted at the prime focus of the Blanco $4 \mathrm{~m}$ telescope at the Cerro-Tololo International Observatory (CTIO). The camera imager is a vacuum vessel that houses the $\mathrm{CCD}^{3}$ array, the focal plane support plate, the Vacuum Interface Board (VIB) and the liquid nitrogen heat exchanger used to cool the CCDs. The CCD array is installed on the front surface of the focal plane support plate. This plate is supported at its outer diameter by four equally spaced bipods. Each bipod leg is anchored to the bipod ring. The bipod ring bolts to a heavy support ring welded to the vacuum vessel outer shell. Flexible copper straps connect the heat exchanger to the back surface of the focal plane support plate to complete the thermal conduction path from the CCDs to the heat exchanger. The imager cooling system ${ }^{4}$ circulates liquid nitrogen through the heat exchanger. An electric heater and Resistance Temperature Detector (RTD) are installed on each of the flexible copper straps. The power to each heater is modulated to control CCD array temperature. A turbo pump is installed on the back cover to initially pump down the imager. The ion pump holds the imager at full vacuum when the imager cooling system is running. The crates which hold the CCD readout electronics ${ }^{5}$, the instrument control system I/O modules, the ion pump controller, the cable feed-throughs, and the other ancillary equipment are attached to the vacuum vessel outer shell. CCD readout cables run from the CCDs to the VIB and then to the CCD readout crates. Optical element $\mathrm{C} 5$ is installed in the $\mathrm{C} 5$ cell. The $\mathrm{C} 5$ cell bolts to the imager front flange and the interface flange to the Barrel.

\section{REQUIREMENTS}

\subsection{Camera Design Requirements}

The camera design requirements ${ }^{6,7}$ that apply to the CCD imager design are listed.

- Expected range of mean focal plane temperature: $-120^{\circ} \mathrm{C}$ to $-80^{\circ} \mathrm{C}$.

- The detector focal plane spatial temperature variation will be $\leq 10^{\circ} \mathrm{C}$ across the focal plane.

- Detector temperature stability will be $\pm 0.25^{\circ} \mathrm{C}$ stability over 12 hours.

\footnotetext{
*Cease@fnal.gov, 1-630-840-6453

${ }^{1}$ Operated by Universities Research Association Inc. under Contract No. DE-AC02-76CH03000 with the United States Department of Energy.
} 
- External surfaces of DECam will be $<3^{\circ} \mathrm{C}$ warmer than ambient temperature.

- All instrument components shall perform to specification at all possible orientations.

- DECam and associated structures will block $<3 \%$ of the light that would otherwise reach the primary mirror.

- Dewar vacuum must be $<2 \times 10^{-4}$ Torr before cooling below ambient.

- Dewar vacuum should be $<10^{-5}$ Torr in normal operation.

- Temperature/vacuum requirements should be met with no manual input more frequently than once per $\sim 30$ hours.

- Vacuum and focal plane temperature should be maintained continuously over a period of $>12$ months without interruption.

- The focal plane should come to operational temperature in $<8$ hours.

- The dewar should warm up and be ready to open to ambient environment in $<12$ hours.

- The dewar and associated cooling and vacuum system will not distort or otherwise move the focal plane by more than $15 \mu \mathrm{m}$ in $\mathrm{x}$ or $\mathrm{y}$.

- The dewar and associated cooling and vacuum system will not distort the flatness of the focal plane by more than $15 \mu \mathrm{m}$.

- The dewar and associated cooling and vacuum system will not vibrate the focal plane by more than $1.5 \mu \mathrm{m}$ at any frequency.

- Detector flatness between adjacent $\leq 1 \mathrm{~cm}^{2}$ areas on a given CCD should be $\leq 10 \mu \mathrm{m}$.

- The optical design shall incorporate a flat focal plane. The peak-to-peak variation in matching CCDs to the focal plane shall be $30 \mu \mathrm{m}$. The positioning of CCDs relative to a flat plane includes any CCD nonflatness.

- Outdoor temperature $-5^{\circ} \mathrm{C}$ to $27^{\circ} \mathrm{C}$.

- Altitude $2200 \mathrm{~m}$ above sea level, $77 \mathrm{kPa}$.

\section{PRIME FOCUS IMAGER DESIGN}

\subsection{Description}

The imager vessel is a 24 inch diameter vacuum vessel that houses the CCD array, the internal cooling system, and the Vacuum Interface Board. The VIB transfers the CCDs's signals from inside the imager to the readout crates. The imager instrument control I/O crates and CCD readout electronics crates are attached to the exterior of the vessel. Three crates house the readout boards for the CCDs. Approximately $2 / 3$ of the CCDs are read out on one side of the imager and the other $1 / 3$ on the opposite side. All of the CCD signals are fed out of the vacuum using a Vacuum Interface Board. The temperature sensors and heater control signals exit the vacuum vessel through a separate fitting near the VIB. The crates are positioned such that each card in the crate can be removed for repair without dismounting the entire crate. Additional instrument control crates are mounted to the imager so that the imager can operate fully while it is not on the telescope. Isometric views of the fully instrumented imager are shown in Figure 1. 


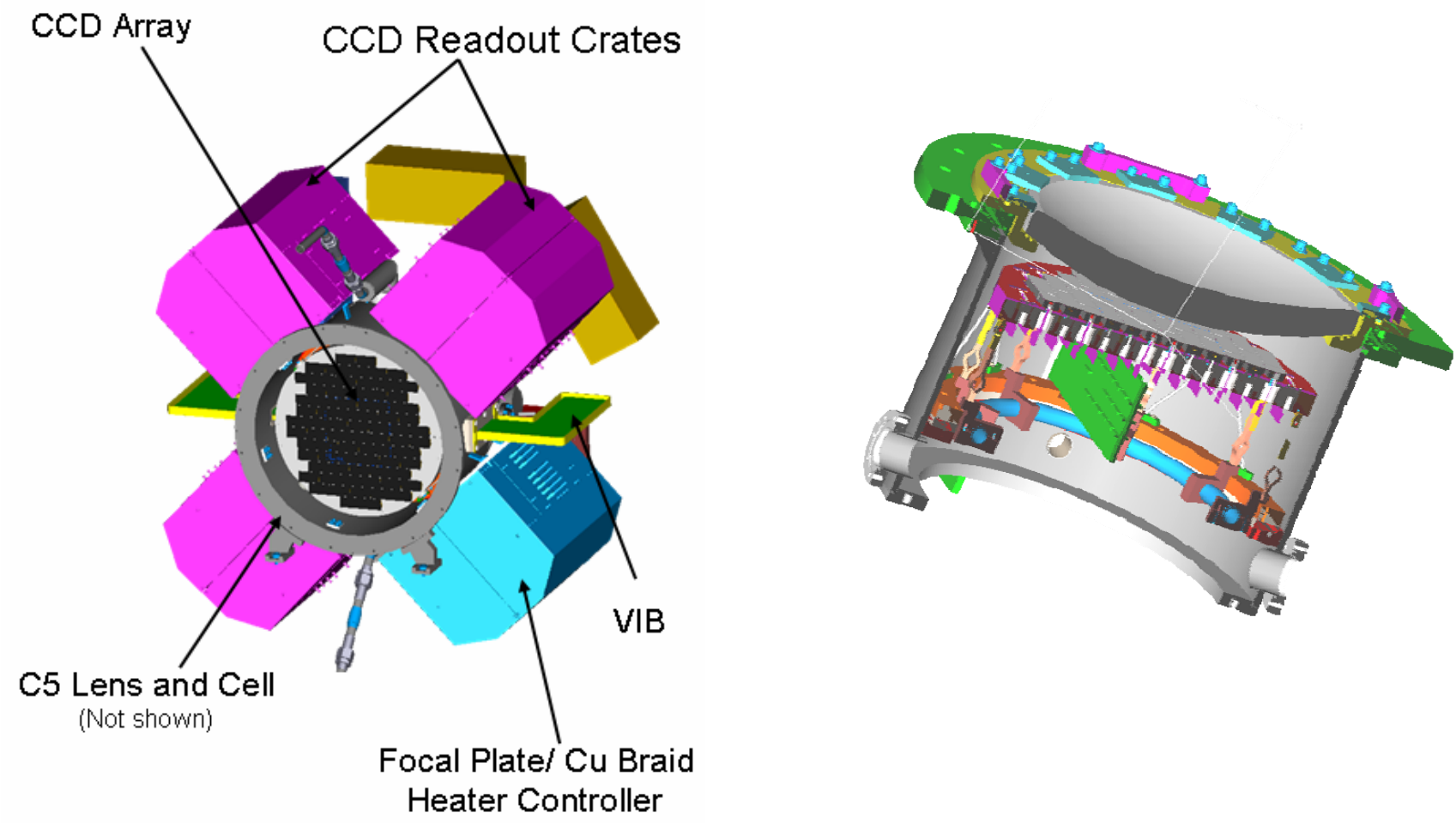

Figure 1. (Top) Isometric view of the fully instrumented imager. (Bottom) Cross section of the imager vessel.

\subsection{Focal Plate Assembly}

The Focal Plate Assembly includes the focal plate which supports all of the CCDs, the bipod supports for the focal plate, and the copper braids for cooling and thermal control. The assembly interfaces with the heat exchanger, the cooling system and the VIB, and is installed by attaching to the internal mounting ring inside the imager vessel. The C5 cell, the rear flange on the imager, and the internal heat exchanger must be removed to gain access to the CCDs. The VIB stays in place. There is an alignment ring between the focal plate assembly and the mounting ring inside the imager vessel. The alignment ring is machined to set the dimension between the CCD array and the C5 optical window. Figure 2 is an illustration of the focal plate assembly.

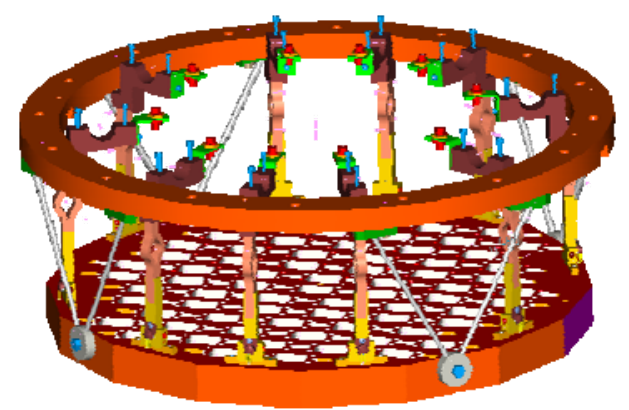

Figure 2. The Focal Plate Assembly, with the heat exchanger and VIB. Isometric view.

\subsubsection{Focal Plate}

The focal plate supports the entire CCD array. The CCD mounting surface must be flat within the budget of the focal plane flatness. The focal plate provides a cold surface for controlling the temperature of the CCDs. The plate is 
constructed of aluminum 6061 tooling plate. Aluminum has good thermal conductivity allowing for small temperature gradients across the focal plate. Aluminum tooling plate is used for its good dimensional stability after machining. The thickness of the plate is sized to minimize the gravitation sag in the plate. The current focal plate geometry is shown in Figure 2. Preliminary Finite Element analysis performed on the focal plate design is shows a focal plate temperature gradient of $1.5^{\circ} \mathrm{C}$ with a flatness within 10 microns.

\subsubsection{Focal Plate Supports}

The focal plate is supported using four sets of bipods. The bipod material is Ti-6Al-4V which is a low thermal expansion, low thermal conductivity, high strength metal. Four bipods are used instead of three due to the symmetry of the CCD array and the VIB. The bipods are all supported off of the bipod support ring to make handling the assembly easier. Final shimming of the focal plate to the $\mathrm{C} 5$ lens can take place between the focal plate assembly mounting ring and the mounting ring inside the CCD vessel. Experience was gained assembling the focal plate when the Multi-CCD Test Vessel was constructed.

\subsubsection{Copper Braids}

The copper braid assembly is the thermal strap between the heat exchanger and the focal plate. The copper braid thickness can be trimmed to adjust the cooling capacity of the braid. A heater and RTD are mounted in the lug on the focal plate end for thermal control. A $55^{\circ} \mathrm{C}$ thermal cutout bi-metallic switch (Cantherm B1205525AEDCC0E) is mounted to the braid and is used in series with each heater for protection against over heating. Electrical isolation is provided between the copper lug and the aluminum mounting block. The aluminum mounting block is used to attach the copper braid assembly to the focal plate. A layer of 50 micron thick adhesive between the copper lug and the aluminum provides the electrical isolation. The wires for the heater and RTD are routed up the side of the copper braid assembly and terminated in a 7 pin connector at the top of the braid. Making the copper braid assembly modular allows for easy replacement of the braid assembly in the event a heater or RTD is damaged.

\subsection{Heat Exchanger}

The heat exchanger inside the imager vessel is used to remove heat from the focal plate. The focal plate operating temperature is $-100^{\circ} \mathrm{C}$. In order to cool the focal plate to $-100^{\circ} \mathrm{C}$, the heat exchanger operating temperature is $-173^{\circ} \mathrm{C}$ and uses liquid nitrogen as the refrigerant. The heat exchanger is a simple 1 inch tube that makes a single loop around the inside of the imager vessel. A mounting lug at the end of the copper braid attaches to the heat exchanger tube to the copper braid. Ten copper braids are mounted around the circumference of the backside of the focal plate for thermal transfer. To gain access to the CCDs, the heat exchanger can be removed from the Imager vessel. The heat exchanger is removed from the Imager by disconnecting the copper braids and the VCR fittings on the tubing and then the assembly is removed through the back of the vessel. 


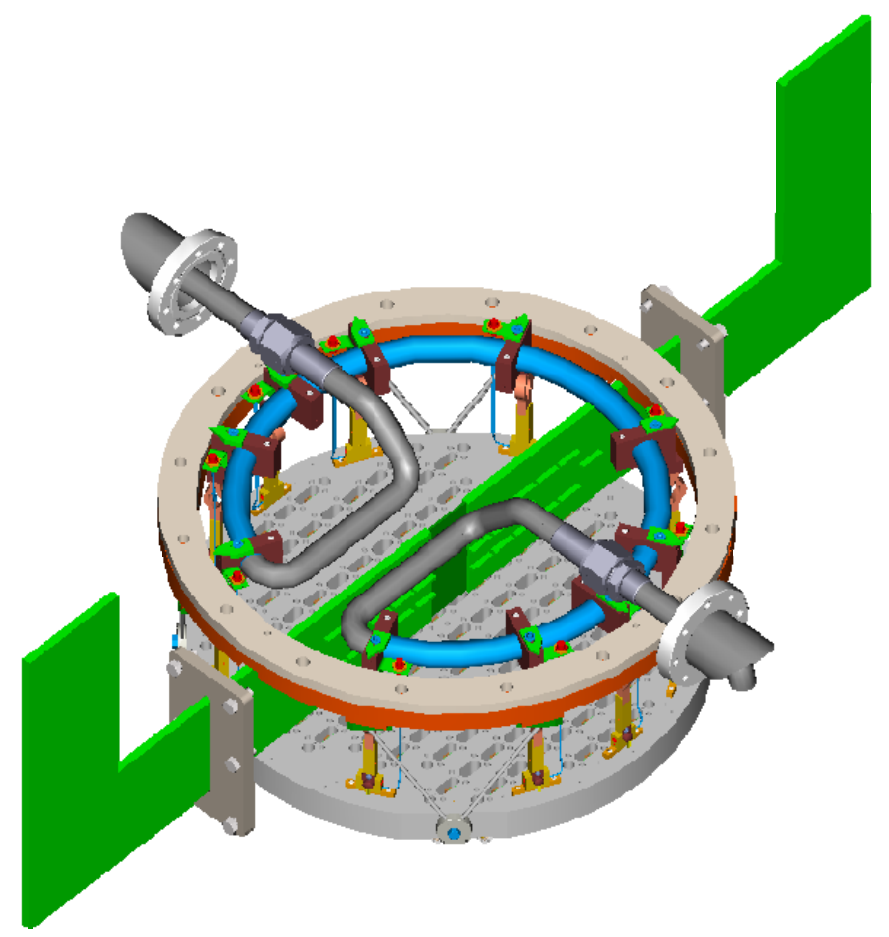

Figure 3. Isometric view of the imager showing all the internal components.

\section{VACUUM SYSTEM}

\subsection{Vacuum Requirements.}

The imager vessel contains the CCD array. The CCDs operate at a temperature that is below the ambient dew point. To prevent water condensation from forming on the CCD array, the imager vessel is evacuated. The initial vacuum in the vessel is achieved by using a turbo pump. The turbo pumping station is too large to ride with the telescope and is only used to maintain vacuum when cooling down or warming up the imager. Once vacuum is achieved with the turbo pump, then the heat exchanger and ion pumps can be used to maintain good vacuum and the turbo pump can be removed from the imager. Since the heat exchanger operates at cryogenic temperatures it is used as an effective cryopump.

During CCD operations the vacuum is required to be below at least $10^{-5}$ Torr in order to prevent water vapor from depositing onto the CCDs. At least $2 \times 10^{-4}$ Torr is required in the imager vessel before the heat exchanger is cooled. The initial vacuum is achieved with a turbo pump and pumping station. Once the focal plate is at the operating temperature, the ion pump is put into operation allowing the large turbo pumping station to be removed from the imager.

\subsection{Gas Loads on the Vacuum System}

Gas loads on the vacuum system are caused primarily from outgassing and permeation through seals. Major components that cause outgassing are the surfaces inside the imager vessel. The imager vessel itself is all stainless steel. The vessel is a 24 inch diameter, 18.75 inch long vacuum vessel. Kapton cables approximately $2 \mathrm{~cm}$ wide by $35 \mathrm{~cm}$ long are used to readout the detectors. The VIB consists of two G-10 boards $13 \mathrm{~cm}$ tall by $35 \mathrm{~cm}$ long. They are used inside the vacuum as an electronic signal feed through board. The front window is fused silica and is $52 \mathrm{~cm}$ in diameter. A second component to the gas load is the permeation rates through the seals used. The window, the window's stainless steel mounting flange, the rear cover, and the vacuum interface board flanges all use o-ring seals. Instrumentation flanges that will not be opened frequently use copper gaskets for the vacuum seal. The total expected gas load from outgassing is $7.5 \times 10^{-4}$ Torr.L/s and is dominated by water vapor outgassing from the Kapton cables. The total expected gas load from permeation is $5 \times 10^{-6}$ Torr.L/sec coming through o-ring type seals. 


\subsection{Vacuum System}

Typical turbo pumping speeds on a 6 inch flange are about $200 \mathrm{~L} / \mathrm{sec}$ (ATH-200 L/sec Hybrid Dry Turbo Pump). An ultimate vessel pressure of $6 \times 10^{-6}$ Torr obtained from using this turbo pump exceeds the requirement for $2 \times 10^{-4}$ Torr before cooling the vessel down, and meets the $10^{-6}$ Torr requirement while starting to warm up without the ion pump and cryopump.

Once the vessel has been pumped down to $6 \times 10^{-6}$ Torr with the turbo pump, a cryopump (minimum cryo pump surface area of $600 \mathrm{~cm}^{2}$ at a temperature less than $160 \mathrm{~K}$ ) and an ion pump (Varian $75 \mathrm{~L} / \mathrm{sec}$ ) will be used to maintain the vessel vacuum pressure. Cryopumping will remove the water and the ion pump will remove the other gases like nitrogen that do not freeze out on the cold surfaces. The majority of the gas load is water vapor. The nitrogen gas load is approximately 100 times less than the overall gas load. To be conservative, a desired vessel vacuum operating pressure of $1 \times 10^{-7}$ Torr is used. A full range vacuum gauge is used to monitor the pressure of the imager vessel. A Pfieffer PKR 251 full range gauge has been selected for this purpose.

All of the vacuum components are mounted to the rear flange of the imager vessel. The vacuum side flange ports have a baffle to prevent light leaks into the vessel and to prevent debris from falling into the vacuum components.

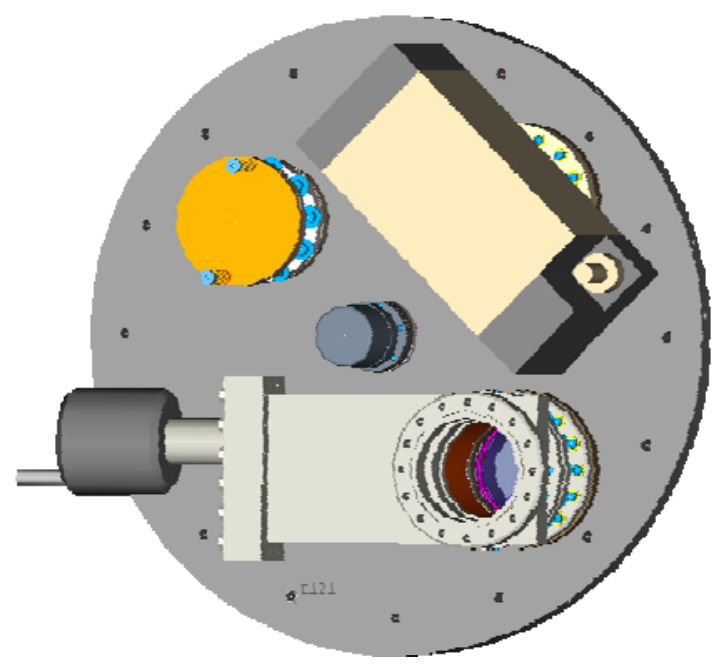

Figure 4. Imager vessel rear flange surface showing gate valve for the turbo pump, the ion pump, the vacuum gauge, and a positive pressure relief mechanism

\section{CELL DESIGN, C5 OPTICAL ELEMENT}

\subsection{C5 Cell Assembly}

The C5 cell must hold the curved lens that is also used as the vacuum window to the instrument dewar. The cell has a spherical surface to match the curvature of the lens where the lens makes contact with the cell. O-rings and o-ring grooves in the spherical surface of the cell make the vacuum seal. When vacuum is not applied to the lens, the lens is held in place by polymer restraints. Four restraints guide the lens radially. The radial restraints are used to center the lens in the cell, and to keep the lens on center when the vacuum load is cycled. Eight axial and radial combined restraints provide enough force to keep the lens in contact with the o-ring seal. After the cell is assembled, it is then aligned to the interface flange. The interface flange is the interface for the barrel, the C5 cell, and the camera vacuum vessel. Figure 5 illustrates the components in the cell and how it is mounted to the camera vessel. 


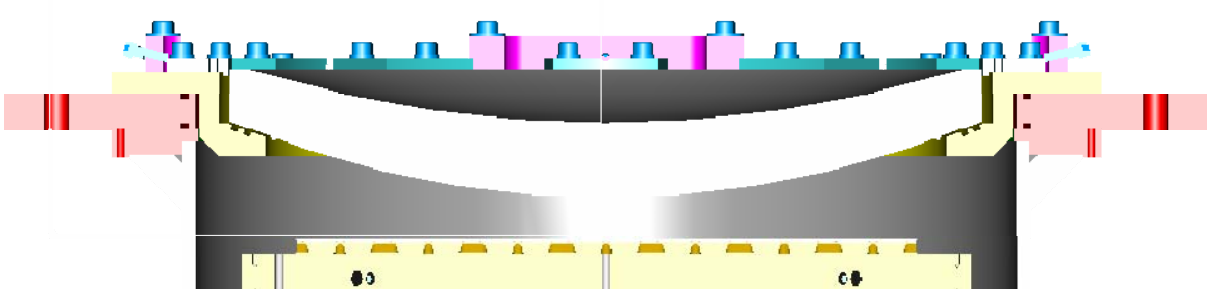

Figure 5. Cross Section of the C5 cell mounted to the prime focus camera vessel.

\subsection{C5 Lens}

The $\mathrm{C} 5$ lens is specified as part of the optical design ${ }^{8}$. The $\mathrm{C} 5$ lens thickness is sized so that it does not distort more than 40 microns from the nominal surface profile under gravity, vacuum and thermal loading. Greater distortions in the lens would influence the optical image. The cell design ensures that lens mounting and vacuum load stresses are at least a factor of 10 less than the yield strength of the lens. The guideline used for stress is Brookhaven's "Guide for Glass and Plastic Window Design for Pressure Vessels, formerly ESH Std. 1.4.2". The tensile yield strength for fused silica is typically $54 \mathrm{MPa}$. The outer radius of the lens is $25 \mathrm{~mm}$ larger than the optical clear radius to provide enough surface area for the vacuum seal.

\subsection{C5 Cell}

The C5 lens is analyzed with the gravity, vacuum and thermal conditions it will be exposed to in operations. The maximum stress calculated in the lens under 1 atmosphere loading, and at operating temperature, is $2.8 \mathrm{MPa}$. The fused silica tensile strength is $54 \mathrm{MPa}$ which is a factor of 19 greater than the calculated stress in the lens. The maximum deflection in the lens calculated is 30 microns. The lens is thermally coupled to the focal plate by thermal radiation. The thermal load is significant and cools the $\mathrm{C} 5$ lens to below freezing at the center of the lens. A dry gas purge is blown in the space between the $\mathrm{C} 4$ and $\mathrm{C} 5$ lenses to help keep the lens warm and to prevent condensation from forming on the $\mathrm{C} 5$ lens.

\subsection{Interface Flange}

The interface flange is used to setup an alignment coordinate system between the imager vessel, the C5 cell, and the corrector body. A coordinate system is setup on the imager vessel using dowel pin holes in the front face of the imager vessel mounting flange. The pins in the vessel flange are then used as a coordinate system to align the focal plate and CCDs. The interface flange is aligned to the corrector body and the imager vessel at Fermilab. The interface flange is then removed and shipped to University College London (UCL). The interface flange with the C5 cell is aligned to the corrector body at UCL. At UCL the C5 cell is centered on the interface flange and aligned with the barrel. The barrel and imager vessel are later joined by re-pinning the interface flange to the imager vessel. The repeatability tolerance in the pinned joints is $+/-12.5$ microns.

\section{IMAGER COOLING SYSTEM AT CTIO}

\subsection{Imager Cooling System}

The imager cooling system contains all of the components required to operate the CCDs at the desired operating temperature. The CCDs are mounted to the focal plate assembly. The focal plate assembly is driven to the operating temperature by the copper braid assembly on the backside of the focal plate. The copper braids couple the focal plate to the cooling heat exchanger and have heaters and RTDs to control the temperature of the back side of the focal plate. The heat exchanger is a stainless steel tube inside the imager vessel that is attached to copper braids. The heat exchanger is supplied with liquid nitrogen by the liquid nitrogen cooling system. The liquid nitrogen system contains all of the components required to supply the imager vessel with liquid nitrogen. A 200 liter dewar that contains a circulation pump and condenser to convert the boil-off gas to liquid is located near the telescope operator control room. Vacuum jacketed transfer lines connect the 200 liter dewar to the imager vessel. The plumbing path is illustrated in Figure 6. 


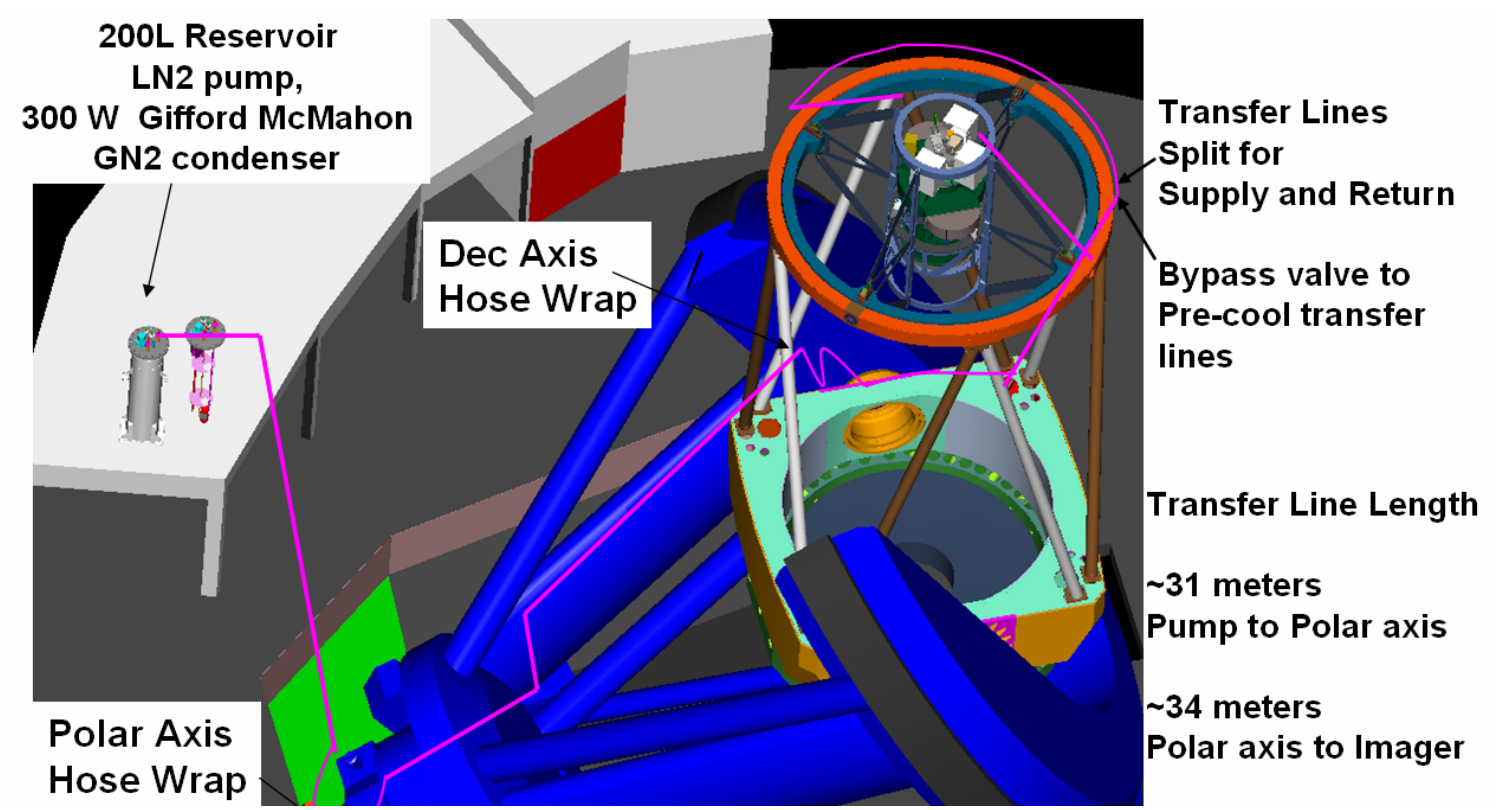

Figure 6. Plumbing path for the imager cooling system.

\subsection{The Thermodynamic and Transport Conditions in the Closed Loop System}

The liquid nitrogen transport conditions and design criteria $^{2}$ used to develop the cooling system are listed below:

1) Pump is seven meters above the floor on top of counting-house. Piping must rise twelve meters higher in order to accommodate for telescope dimensions and position.

2) Operating at $0.76 \mathrm{MPa}$, or 100 psig with 11 psi atmospheric pressure on top of the mountain. Liquid temperature $100 \mathrm{~K}$.

3) Fluid flow is 110 grams/sec $\sim 2$ gpm

4) Telescope position is both vertical (at zenith) and horizontal (at horizon)

A schematic of the cooling system is shown in Figure. The coolant properties at each of the numbered locations have been calculated.

The fluid is sub-cooled or is single phase at all points except points 3.5, 4 and 5. Points 4 and 5 are located at the heat exchanger inside the imager. At the imager two phase flow is expected since the cooling is provided by evaporating liquid nitrogen in the heat exchanger. The flow rate and pressure is selected to ensure that evaporated nitrogen generates small bubbles that are entrained in the fluid flow. 


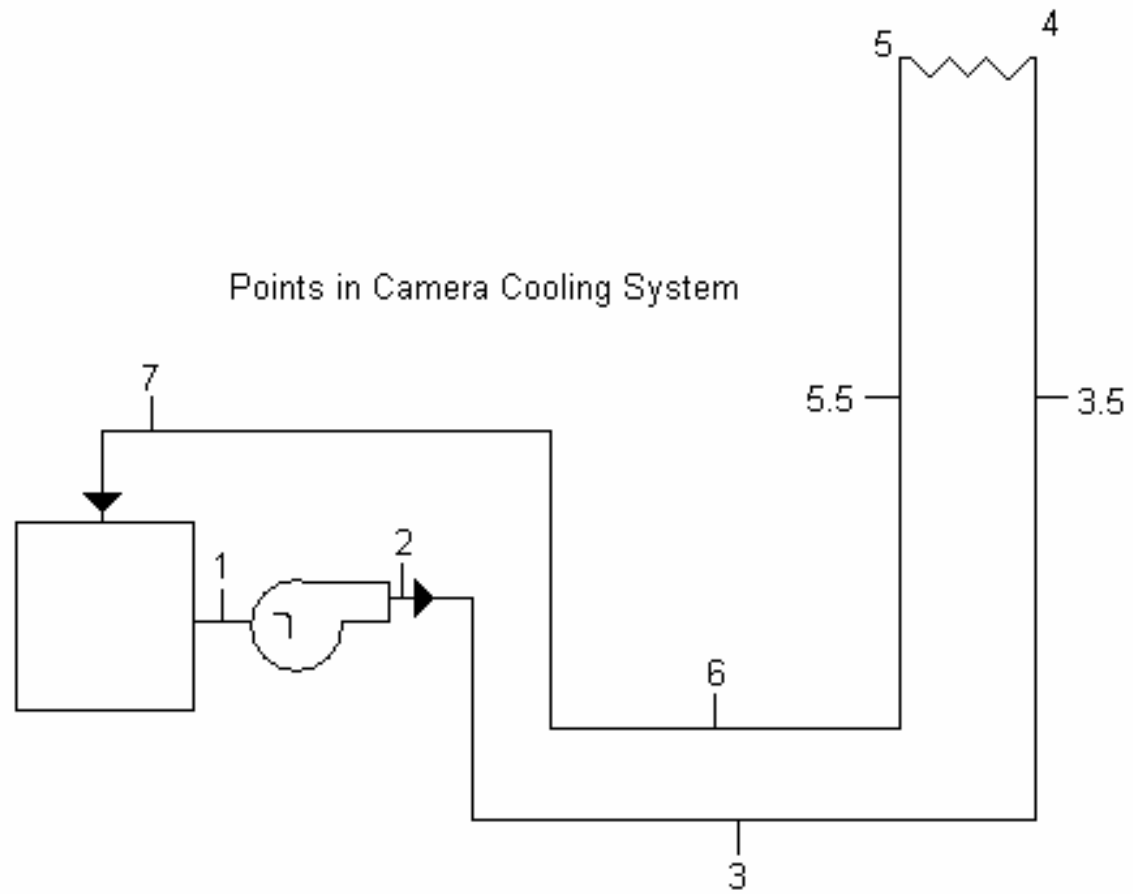

Figure 7. Schematic of the cooling system

\subsection{Heat Loads}

The heat loads are from the imager vessel, the transfer lines, and the loads in the 200 Liter reservoir. The heat loads due to the imager vessel are summarized in Table 1. The calculated load in the imager vessel is 113 Watts in the nominal steady state case.

Table 1. Cooling System heat loads.

\begin{tabular}{|l|l|}
\hline Item & Heat load budget \\
\hline Imager Vessel & 113 watts \\
\hline Transfer Lines & 65 watts \\
\hline Valves, fittings & 16 watts \\
\hline Circulation pump & 38 watts \\
\hline 2001 Reservoir & 25 watts \\
\hline Total & 257 watts \\
\hline
\end{tabular}

The cryo-cooler used to condense the returning gaseous nitrogen has a capacity of 360 watts at an operating temperature of $90 \mathrm{~K}$. The cryo-cooler has $40 \%$ more capacity than what is required in the steady state operating condition.

\section{PROTOTYPE MULTI-CCD TEST VESSEL}

\subsection{Multi-CCD Test Vessel Description}

The Multi-CCD Test Vessel is a full sized prototype camera vessel. It provides the test bed for multi CCD readout in an environment very close to what is imagined for the final CCD imager vessel design. It has a flat optical window and cell similar in thickness to the $\mathrm{C} 5$ lens and the $\mathrm{C} 5$ cell. At this time $10 \mathrm{CCDs}$ have been mounted to the focal plane support 
plate. The initial experiences in cooling the focal plate to the desired operating temperature are presented. Flatness measurements on the 10 CCD mosaic when cold are also presented.

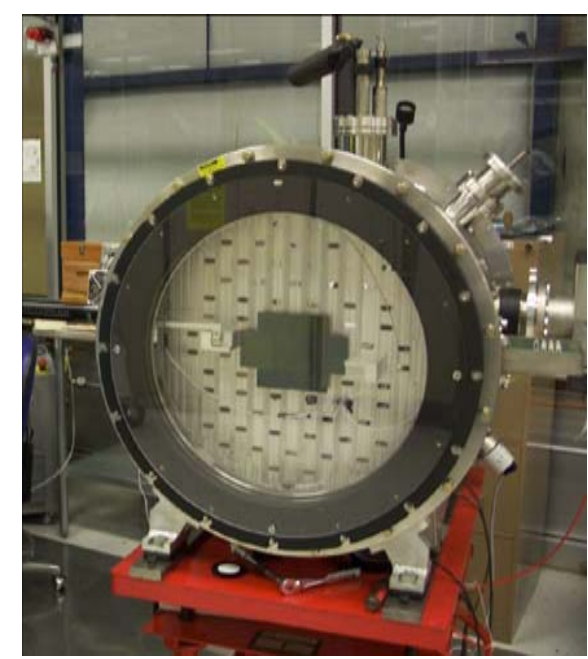

Figure 8. Multi-CCD Test Vessel with the $10 \mathrm{CCD}$ engineering grade mosaic.

\subsection{Temperature Tests}

The Multi-CCD Test vessel has a cooling system and internal heat exchanger that is very similar to the final version. The temperature control system is tested using this system. Heaters in the copper braids are controlled using a cascade PID control system. The temperature sensor in the copper braid is used as the first step in the cascade PID loop and a temperature sensor very near the CCDs is used as the second step in the cascade PID loop. By using a cascade system, the heaters in the braid can react more quickly to temperature upsets or environmental temperature changes occurring at the front of the focal plate. The cascade loop has allowed the temperature at the front of the focal plate to be stable within the operating requirements of $\pm 0.25^{\circ} \mathrm{C}$. An example of the cool down curve on the Multi-CCD test vessel is shown in Figure 9.

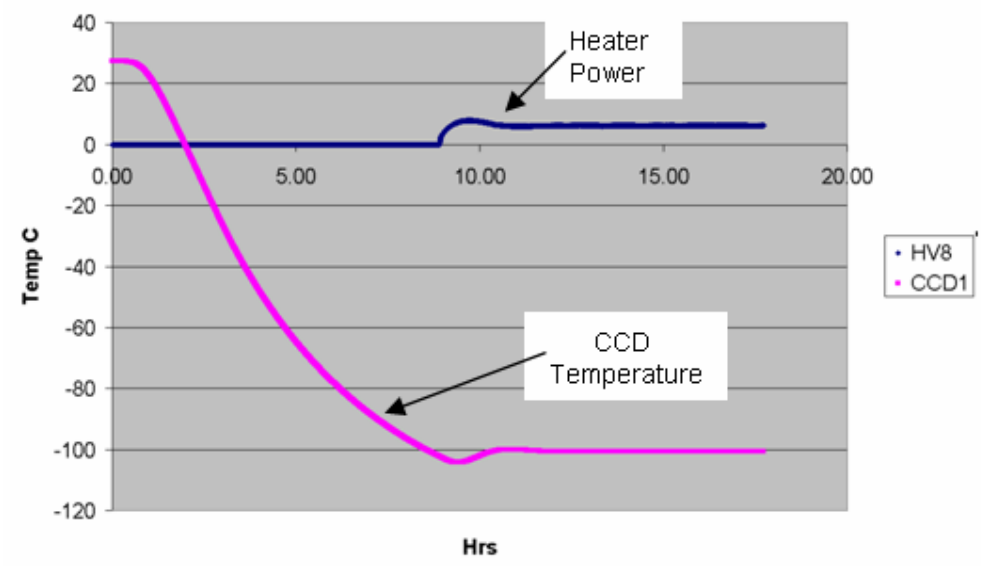

Figure 9. Example Focal Plane Cool Down Rate in the Multi- CCD Test Vessel. 


\subsection{Focal Plate Flatness Measurements}

Flatness of the focal plate can be measured both when the CCD mosaic is warm and cold through the flat optical window. The measurement is taken using the Micro-Epsilon Opto-NCDT 2400 confocal measuring device. The measuring head is mounted to a set of precision X-Y slides and is readout and controlled using a National Instruments Labview program. The setup is mounted to the front of the Multi-CCD Test Vessel shown in Figure 10. Initial flatness data taken over the $10 \mathrm{CCD}$ mosaic at $-100^{\circ} \mathrm{C}$ is shown in Figure 11.

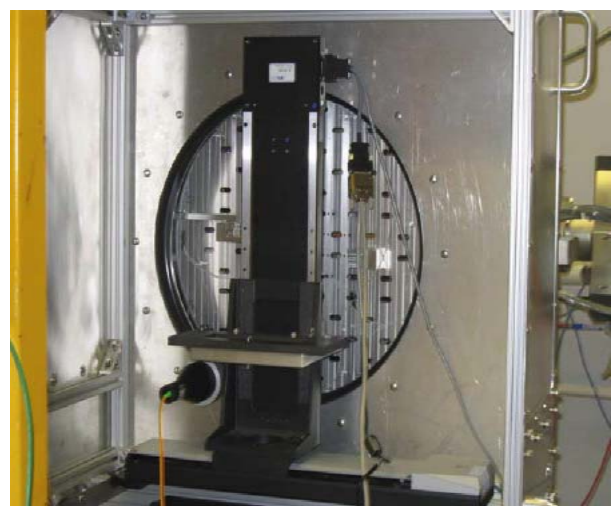

Figure 10. Flatness measurement setup mounted to the front of the Multi-CCD Test Vessel.

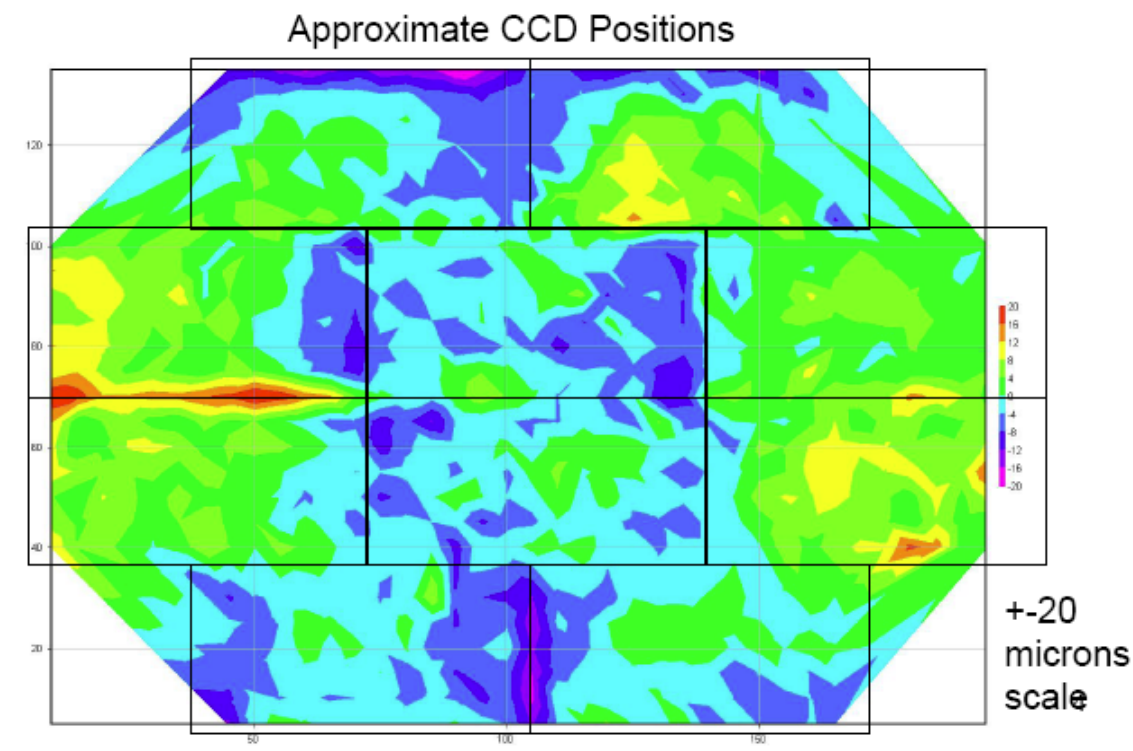

Figure 11. Preliminary flatness measurement of the $10 \mathrm{CCD}$ engineering grade mosaic.

\section{CONCLUSION}

The imager design is fully developed and provides an environment suitable for the CCD array. A prototype of the imager vessel has been manufactured and is used for Multi-CCD testing. CCD testing and operations have been performed in the 
prototype vessel successfully. Operating requirements for vacuum, temperature stability, and focal plate flatness have all been verified using the Multi-CCD test vessel.

\section{ACKNOWLEDGEMENTS}

Funding for the DES Projects has been provided by the U.S. Department of Energy, the U.S. National Science Foundation, the Ministry of Science and Education of Spain, the Science and Technology Facilities Council of the United Kingdom, the National Center for Supercomputing Applications at the University of Illinois at UrbanaChampaign, the Kavli Institute of Cosmological Physics at the University of Chicago, Financiadora de Estudos e Projetos, Fundação Carlos Chagas Filho de Amparo à Pesquisa do Estado do Rio de Janeiro, Conselho Nacional de Desenvolvimento Científico e Tecnológico and the Ministério da Ciência e Tecnologia and the Collaborating Institutions in the Dark Energy Survey.

This work has taken place with the help of all of the Collaborating Institutions. The Collaborating Institutions are Argonne National Laboratories, the University of Cambridge, Centro de Investigaciones Energeticas, Medioambientales y Tecnologicas-Madrid, the University of Chicago, University College London, DES-Brazil, Fermilab, the University of Edinburgh, the University of Illinois at Urbana-Champaign, the Institut de Ciencies de l'Espai (IEEC/CSIC), the Institut de Fisica d'Altes Energies, the Lawrence Berkeley National Laboratory, the University of Michigan, the National Optical Astronomy Observatory, the Ohio State University, the University of Pennsylvania, the University of Portsmouth and the University of Sussex.

\section{REFERENCES}

${ }^{[1]}$ D. DePoy et al, "The Dark Energy Camera (DECam)" in these proceedings.

${ }^{[2]}$ B. Flaugher, “The Dark Energy Survey”, Int. J. Modern. Phys. A20, 3121 (2005)

${ }^{[3]}$ Greg Derylo, H. Thomas Diehl, Juan Estrada, "0.25 mm-Thick CCD Packaging for the Dark Energy Survey Camera Array”, High Energy, Optical, and Infrared Detectors for Astronomy II. Edited by Dorn, David A.; Holland, Andrew D., Proceedings of the SPIE, Volume 6276, 627608 (2006).

${ }^{[4]}$ R.L. Schmitt et al, “Cooling the Dark Energy Camera Instrument”, SPIE 7014-243 in these proceedings.

${ }^{[5]}$ L. Cardiel, "FEE for DECam", et al, Conf 7014-244 in these proceedings

${ }^{[6]}$ Abbott, T., Annis, J., DePoy, D.L., Flaugher, B., Kent, S., Lin, H., Merritt, W., "Dark Energy Camera Specifications and Technical Requirements", 10 June 2008

${ }^{[7]}$ Stefanik, A., "Environmental Design Conditions", August 29, 2007.

${ }^{[8]}$ DECam optics and alignment P. Doel et al, Conf 7014-65 in these proceedings. 